\title{
Polyurethane foam coated with a multi-walled carbon nanotube/polyaniline nanocomposite for a skin-like stretchable array of multi-functional sensors
}

\author{
Soo Yeong Hong ${ }^{1}$, Ju Hyun $\mathrm{Oh}^{1}$, Heun Park ${ }^{1}$, Jun Yeong Yun ${ }^{1}$, Sang Woo Jin ${ }^{2}$, Lianfang Sun ${ }^{3}$, Goangseup Zi ${ }^{3}$ \\ and Jeong Sook $\mathrm{Ha}^{1,2}$ \\ Body-attachable sensors can be applied to electronic skin (e-skin) as well as safety forewarning and health monitoring systems. \\ However, achieving facile fabrication of high-performance, cost-effective sensors with mechanical stability in response to \\ deformation due to body movement is challenging. Herein we report the material design, fabrication and characteristics of a \\ skin-like stretchable array of multi-functional (MF) sensors based on a single sensing material of polyurethane foam coated with \\ multi-walled carbon nanotube/polyaniline nanocomposite, which enables simultaneous detection of body temperature, wrist pulse \\ and ammonia gas. These sensors exhibit high sensitivity, fast response and excellent durability. Furthermore, the fabricated \\ sensor array shows stable performance under biaxial stretching up to $50 \%$ and attachment to skin owing to the use of direct- \\ printed Galinstan liquid metal interconnections. This work proposes a facile method for fabrication of high-performance, \\ stretchable MF sensors via appropriate selection of sensor design and functional materials that are applicable to e-skin and \\ health monitoring systems.
}

NPG Asia Materials (2017) 9, e448; doi:10.1038/am.2017.194; published online 17 November 2017

\section{INTRODUCTION}

Wearable electronics have attracted considerable attention for use in electronic skin (e-skin) and health monitoring systems in order for a comfortable and secure life. ${ }^{1-5}$ e-Skin is a human interactive device that can simultaneously sense signals from the body and respond to the environment. Thus it should be capable of measuring the five types of senses (taste, sight, hearing, olfactory and touch sensation) with high sensitivity and show mechanical stability against deformation due to skin movement. As a result, e-skin is required to be very thin and have a strain-relaxed design. ${ }^{6}$

Because of the increasing importance of e-skin, extensive efforts have been undertaken to develop various sensors with high sensitivity. ${ }^{7}$ Beyond the conventional sensor that can detect a single stimulus, novel advanced sensors for simultaneous monitoring of multiple stimuli (multi-functional (MF) sensors) have been actively investigated. ${ }^{8,9}$ For practical application of such MF sensors, it is necessary to eliminate the interference between different stimuli that is commonly observed in conventional MF sensors, ${ }^{10}$ in addition to fabricating cost-effective sensors on a deformable substrate. Development of MF sensors that transduce different stimuli into separate signals can intrinsically minimize signal interference, thus allowing for sensitive detection of multiple parameters, such as temperature and pressure, in a single device without decoupling analysis. Many power- generation devices, such as solar cells and a readable signal in addition to the flowing current. ${ }^{11}$

Among the various available sensor materials (multi-walled carbon nanotubes (MWCNTs), graphene, metal oxides, and conducting polymers), MWCNTs are considered to be an ideal candidate owing to their electrical conductivity, large surface area and environmental stability. ${ }^{11,12}$ However, MWCNTs suffer from poor thermoelectric properties and poor gas-sensing performance, which limit their practical applications. ${ }^{13,14}$ To overcome such drawbacks, MWCNTs have been used with metallic nanowires or with conducting polymers in a composite. ${ }^{11}$ Polyaniline (PANI) is considered one of the most promising and widely applied sensing materials because of its low production cost, environmental stability and acceptable conductance as well as its unique ammonia $\left(\mathrm{NH}_{3}\right)$ gas-sensing ability. ${ }^{15}$ In addition, PANI has thermoelectric properties that allow it to exhibit output voltage according to a change in temperature. Furthermore, MWCNTPANI nanocomposites have shown superior performance in terms of the Seebeck coefficient, electrical conductivity and thermal conductivity compared with their individual components. ${ }^{16,17}$

As an excellent alternative candidate to overcome the narrow pressure range and low sensitivity of thin film-based pressure and gas sensors consisting of nanowires, nanoparticle and 3-dimensionally structured electrodes, ${ }^{18-20}$ conductive porous foams are expected to be promising owing to their electronic conductivity and mechanical

${ }^{1}$ Department of Chemical and Biological Engineering, Korea University, Seoul, Republic of Korea; ${ }^{2}$ KU-KIST Graduate School of Converging Science and Technology, Korea University, Seoul, Republic of Korea and ${ }^{3}$ Department of Civil, Environmental and Architectural Engineering, Korea University, Seoul, Republic of Korea Correspondence: Professor JS Ha, Department of Chemical and Biological Engineering, Korea University, 5-1 ka, Anam-dong, Sungbuk-ku, Seoul 02453, Republic of Korea. E-mail: jeongsha@korea.ac.kr 
a
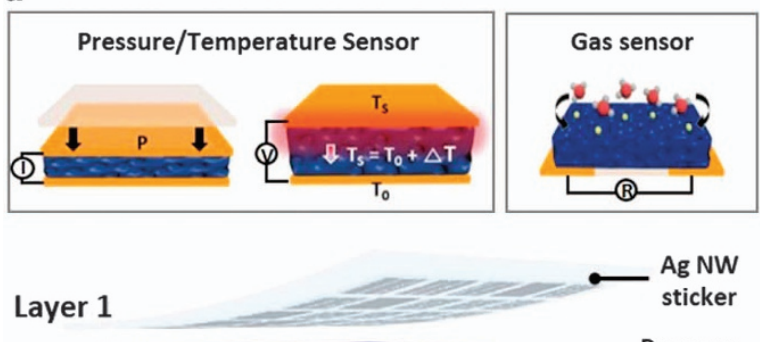

Layer 2

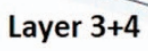

\section{$3+4$}
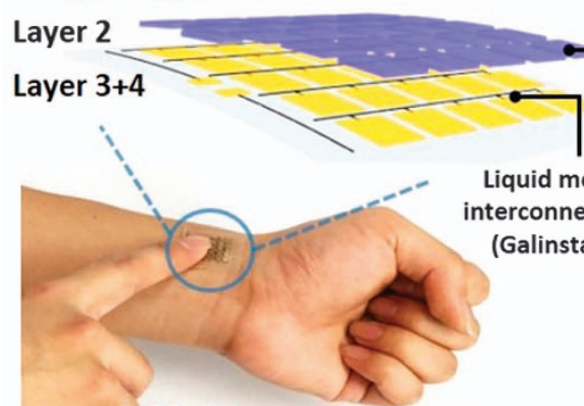

Stretchable bio-environmental sensor array

C (Galinstan) b

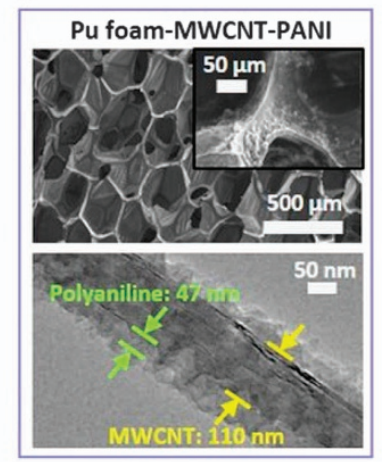

Temperature, Gas sensor array

d
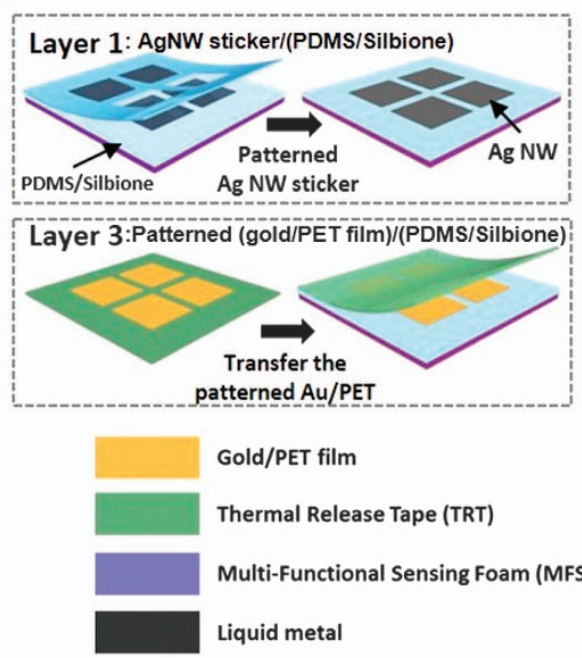

Gold/PET film

Thermal Release Tape (TRT)

Multi-Functional Sensing Foam (MFSF)
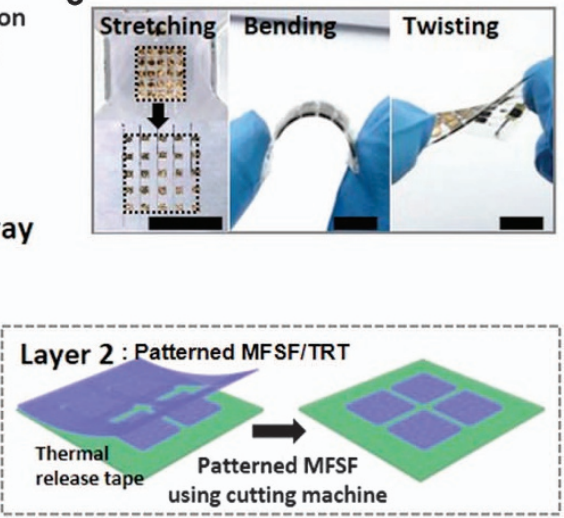

Liquid metal

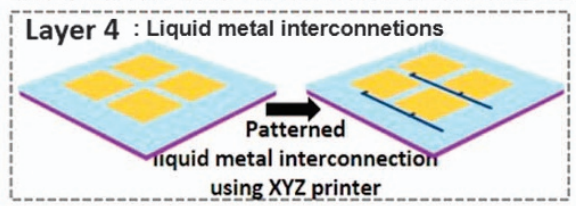

using XYZ printer

Figure 1 (a) Schematic illustration of the skin-attached multi-functional (MF) sensor array with pressure/temperature and gas sensors, integrated using directly printed liquid metal interconnections. (b) (Top) SEM image of the PU foam coated with MWCNT-PANI composite with a zoomed image in the inset. (Bottom) TEM image of the MWCNT-PANI nanocomposite. (c) Optical images of the stretchable multi-functional sensor array in stretched, bent and twisted states, respectively, from left to right. The scale bar is $2 \mathrm{~cm}$. (d) Schematic of fabrication process for the stretchable MF sensor array.

flexibility. ${ }^{21}$ Thus porous foams coated with conductive nanomaterials can be used for fabrication of high-performance pressure, temperature and gas sensors owing to their combined advantages of electronic conductivity, mechanical flexibility and large specific surface area. $^{22}$

Here, for the first time, we demonstrate a skin-like stretchable array of MF sensors based on a single sensing material comprising polymer foam coated with MWCNT-PANI, which enables simultaneous detection of bio-signals such as skin temperature, wrist pulse and ammonia gas for health monitoring when attached to the skin. The pressure sensor shows a wide sensing range from 0.05 to $30 \mathrm{kPa}$, a fast response time of $20 \mathrm{~ms}$, a high sensitivity of $2.1 \mathrm{kPa}^{-1}$ and high stability over 10000 loading/unloading cycles. Owing to the thermoelectric properties of MWCNT-PANI, temperature is measured as an output voltage under a temperature gradient, with a high sensitivity of
$28.7 \mu \mathrm{V}^{\circ} \mathrm{C}^{-1}$. The ammonia gas sensor has a sensitivity of $46.9 \%$, with a response time and recovery time of 7.8 and $34 \mathrm{~s}$, respectively, at 25 p.p.m. To achieve mechanical stability under externally applied strain, polyethylene terephthalate (PET) films are locally implanted on a soft mixed thin film of Silbione and polydimethylsiloxane (PDMS), and then the MF sensors are positioned on top. The sensors are electrically connected via embedded interconnections of the liquid metal Galinstan (eutectic alloy of Ga (68.5\%), In (21.5\%) and Sn $(10 \%)$ ) patterned using a home-built XYZ printer. Furthermore, the integrated MF-sensing foam (MFSF) on the stretchable substrate is easily fabricated into a $5 \times 5$ sensor array using a cut-and-paste method. The stretchable MF sensor array shows mechanical stability under deformations, such as bending, twisting and biaxial stretching up to $50 \%$, and demonstrates no deterioration during the resultant spatial distribution in pressure, temperature and gas sensing. 


\section{MATERIALS AND METHODS}

\section{Fabrication of stretchable MF sensor array}

Our stretchable MF sensor array with a multilayered structure was fabricated by assembling the four component layers (that is, Layer 1, Layer 2, Layer 3 and Layer 4). To make 'Layer 1', a mixture of Silbione and PDMS in a 9:1 ratio was spin-coated on glass at 1000 r.p.m. for $1 \mathrm{~min}$ and cured in a dry oven at $65^{\circ} \mathrm{C}$ for $10 \mathrm{~min}$. After attachment of the patterned PET film (thickness: $100 \mu \mathrm{m}$ ), a silver nanowire (Ag NW) solution (1 wt\% diluted in water, $65 \mathrm{~nm}$ average diameter, $10 \mu \mathrm{m}$ average length, Ditto Technologies, Gunpo, Korea) was dropped onto an empty area of the PET film and dried at room temperature. Scanning electron microscopic (SEM) images of the Ag NW sticker are displayed in Supplementary Figure S3. Thus the Ag NW sticker can be attached between other layers and active devices for stable electrical contact. To make 'Layer 2', the MFSF was attached to thermal release tape (TRT) and adhered to a sticky cutting mat. The MFSF on TRT can be cut automatically using a cutting machine (Silhouette, Lindon, UT, USA). ${ }^{23,24}$ The excess MFSF segment was detached from the TRT using tweezers. Then it was peeled from the cutting mat. To make 'Layer 3', Au/Ti (50/5 nm)-deposited PET film was patterned on TRT using a cutting machine. This TRT film was transferred onto a mixed substrate of Silbione and PDMS.

Layer 2 was attached to Layer 1, followed by slight baking of these films on a $90{ }^{\circ} \mathrm{C}$ hotplate to deactivate the adhesives on the TRT. As a result, the excess adhesive can be easily peeled off with tweezers. Layer 3 was fabricated in the same manner. After assembling those three layers, Layer 4 was assembled to create electrical interconnections. The thickness of the entire substrate is $1 \mathrm{~mm}$.

\section{RESULTS AND DISCUSSION}

A schematic illustration of a skin-attached, stretchable array of MF sensors is shown in Figure 1. With this sensor array, ammonia gas and body temperature and wrist pressure bio-signals can be simultaneously monitored. The whole system, consisting of four stacked layers, contains an array of pressure/temperature and gas sensors fabricated using a single sensing material of MWCNT-PANI nanocompositecoated polyurethane (PU) foam as shown in Figure 1a. The SEM image in Figure 1b (top) confirms a high density of micropores and a large surface area in the PU foam. The inset shows that MWCNTPANI nanocomposites fully cover the PU foam surface. The transmission electron microscopic image in Figure $1 \mathrm{~b}$ (bottom) also confirms that a highly uniform and thick PANI layer covers the MWCNT surface, thus providing stable performance of the composite-based sensor devices. The thickness of the polymer layer on the MWCNT was estimated to be approximately $47 \mathrm{~nm}$. The diameter of the MWCNTs was observed to be $110 \mathrm{~nm}$. More detailed optical images and SEM images of MWCNTs, PANI and MWCNT-PANI are shown in Supplementary Figures S1-S3. Raman spectra of the MWCNTs and MWCNT-PANI nanocomposites are shown in Supplementary Figure S4. The typical G-band corresponding to the $\mathrm{sp}^{2}$ vibration of the hexagonal lattice in the graphite appears at $1575 \mathrm{~cm}^{-1}$ in the spectrum of the bare MWCNTs. The bands at 1347 and $2676 \mathrm{~cm}^{-1}$ correspond to the disordered graphitic structure (D-band) and second-order graphitic structure (D-band), respectively. After polymerization of MWCNTs with PANI, additional peaks that are characteristic of protonated PANI appear at 1170 and $1504 \mathrm{~cm}^{-1} .25,26$ The fabricated sensor array is conformally integrated on the skin and is mechanically stable under stretching as well as bending and twisting deformations (Figure 1c). Figure 1d shows the fabrication of the four layers. A mixture of Silbione and PDMS in a 9:1 ratio was spin-coated on glass and cured for the sensor array, which is skin-attachable without the use of glue. After attachment of the patterned PET film on the PDMS/Silbione substrate, a Ag NW solution was dropped onto the open area of the PET film and dried at room temperature (Layer 1). Using a cutting machine, the MFSF and $\mathrm{Au} / \mathrm{Ti}$ film deposited on the PET film was cut to be automatically patterned on thermal release tape for Layer 2 and Layer 3, respectively. By directly printing liquid metal Galinstan with a home-built XYZ printer, the stretchable interconnections were fabricated over a large area on a spin-coated mixture of Silbione/PDMS substrate (Layer 4). The detailed fabrication of the stretchable substrate is described in Supplementary Figure S5 and Experimental methods section.

In our previous study, stretchable liquid-metal interconnections were realized by injecting Galinstan into polymer-embedded microchannels using a syringe. ${ }^{27,28}$ However, it is difficult to obtain stable interconnections between individual devices via the injection method over a large area. Thus, in this work, we adopted a direct printing method using our XYZ printer with the computer software (Auto$\mathrm{CAD}$ ), which enabled facile and simple fabrication of stretchable Galinstan interconnections over a large area. This printing method exploits the good wettability of Galinstan with the surface of mixed thin Silbione and PDMS film, which is a silicon-based substrate. ${ }^{29,30}$ The sticky property of the film enhances the wetting of the liquid metal on the substrate surface. ${ }^{31}$ Furthermore, formation of the gallium-oxide skin during the direct printing process allows the written pattern to maintain its printed shape, even after the encapsulation process with elastomer. The mechanical stability of the Galinstan interconnection was confirmed by the fact that there was no noticeable change in resistance under stretching up to $50 \%$, and more detailed characteristics of the liquid metal pattern obtained using the XYZ printer are also illustrated in Supplementary Figure S6. As shown in Supplementary Figure S6d, the flux of the liquid metal is controlled through a syringe pump, while the stage develops a predetermined geometry at a controlled velocity $(v)$ under a fixed syringe needle having a particular inner diameter (ID) maintained at a standoff distance $(\approx \pm 5 \mu \mathrm{m})$ from the substrate. We made changes to relieve geometric constraints and a pressure increase that previously occurred during filling of the microchannels. The width measurements of Galinstan were obtained in order to characterize the effects of the system inputs (flux of Galinstan, velocity of patterning, ID of the needle). The results of these measurements are demonstrated in Supplementary Figure S6a-c. The electrical resistance of the patterned Galinstan interconnection created with the XYZ printer was measured while varying the applied uniaxial strain. As shown in Supplementary Figure S6e and f, the normalized resistance, after 1000 repetitions, did not change with uniaxial stretching to $50 \%$ (ID of $514 \mu \mathrm{m}$, flux of $1.5 \mathrm{ml} \mathrm{h}^{-1}$, velocity of $\left.100 \mathrm{~mm} / \mathrm{min}^{-1}\right)$, thus confirming the mechanical stability of the embedded interconnections.

For simultaneous monitoring of pressure and temperature using a single sensor, that is, an MF sensor, we designed a sandwich-type sensor consisting of top and bottom electrodes and a sensing material of PU foam coated with MWCNT-PANI nanocomposite.

Figure $2 \mathrm{a}$ outlines the pressure-sensing mechanism for the fabricated MF sensor. As marked by circles 1, 2 and 3, the frame of the PU foam is in close contact with the adjacent frame and the electrodes (top Ag or bottom $\mathrm{Au}$ ). With an increase in pressure, there will be a change in current flow due to the change in the contact resistance between adjacent PU foam frames coated with the MWCNT-PANI nanocomposite. In addition, the change in the resistance from the reduced thickness of the sensor would lead to a change in current flow.

The pressure sensor exhibits markedly enhanced current with an increase in pressure over the range from 0.05 to $30 \mathrm{kPa}$. The pressure sensitivity $(S)$ is defined as $S=\left(\Delta I / I_{0}\right) / \Delta P$, where $\Delta I$ is the pressureinduced change in current, $I_{0}$ is the initial current flowing across the sensor without pressure loading, and $\Delta P$ is the change in the applied pressure. $S$ is estimated to be $2.1 \mathrm{kPa}^{-1}$ from the linear least-squares 
a

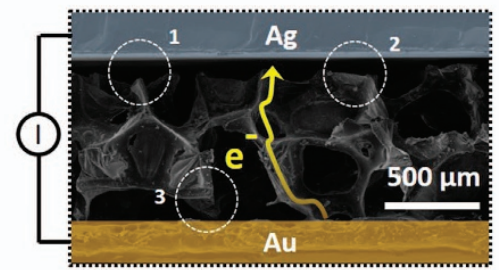

b

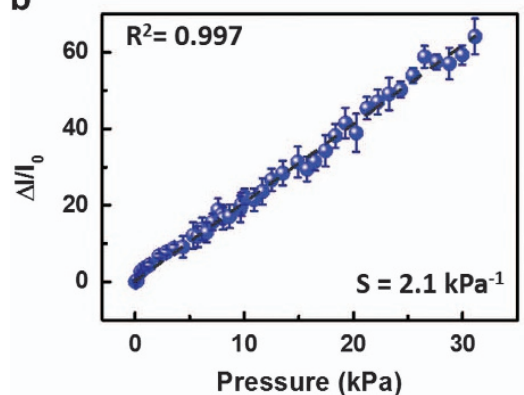

e

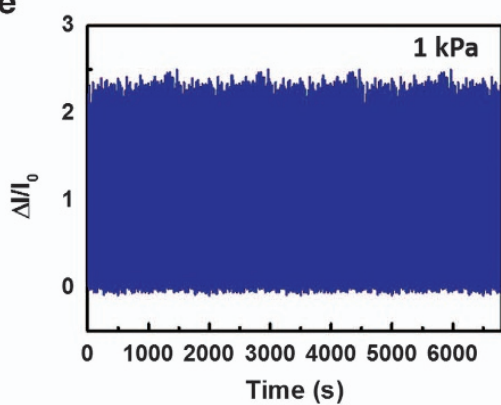

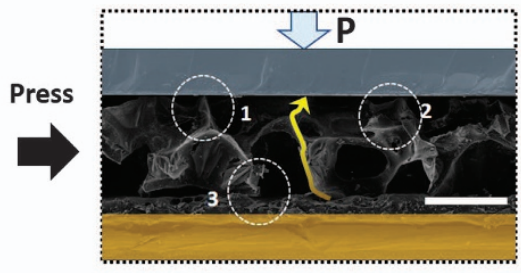

c

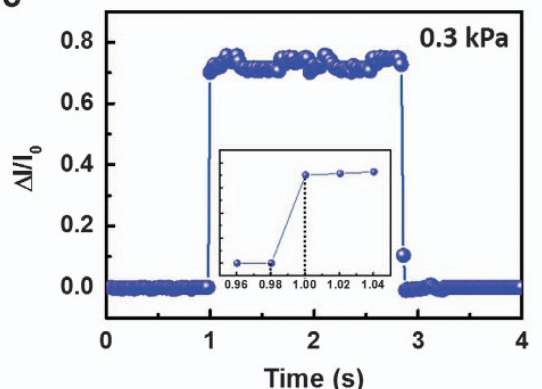

f

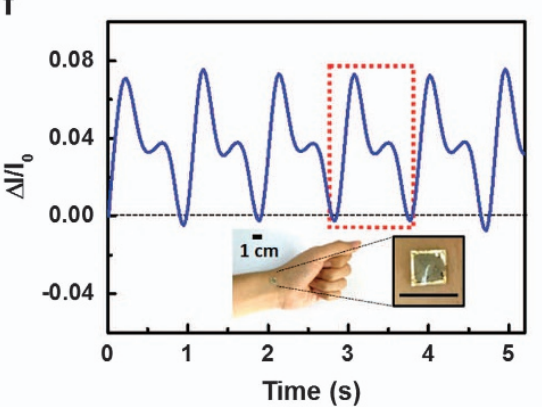

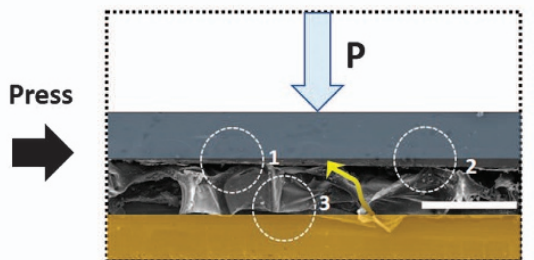

d

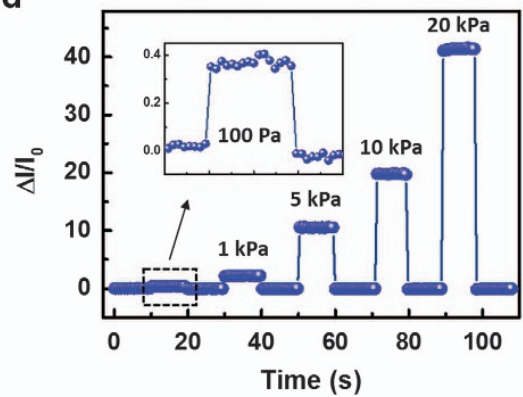

9

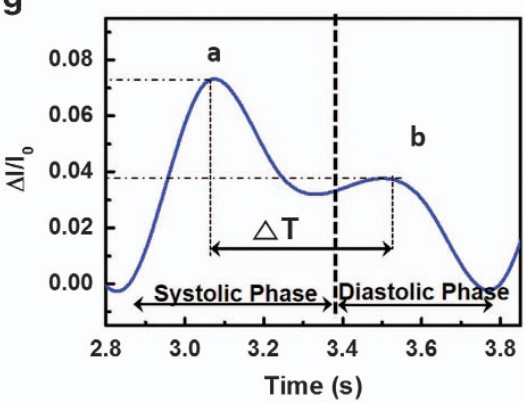

Figure 2 (a) SEM images of the pressure sensor taken with increasing applied pressure. (b) Relative current change ( $\left.\Delta / / I_{0}\right)$ with pressure. (c) Response curve with an applied pressure of $300 \mathrm{~Pa}$, showing a response time of $20 \mathrm{~ms}$. (d) Repetitive measurements of $\Delta / / I_{0}$ with a sequential increase in the pressure from 0.1 to $20 \mathrm{kPa}$. (e) $\Delta / / I_{0}$ under loading/unloading at a pressure of $1 \mathrm{kPa}$ over 10000 cycles. (f) Measurement of real-time wrist pulse waves. (g) The pulse waveform extracted from panel (f), showing some features essential for health monitoring. Here $\Delta /$ and $I_{0}$ are the change in current with application of pressure and the current without applied pressure, respectively.

fitting shown in Figure 2b. The small error bars are the s.d. corresponding to data obtained with five different sensors, showing very high reproducibility. Such a high sensitivity is attributed to the porous microstructure of the $\mathrm{PU}$ foam. ${ }^{10}$ The sensitivity of the pressure sensor based on the bare MWCNTs is similar to that of the MWCNT-PANI nanocomposite, as shown in Supplementary Figure S7a, confirming the advantage of using porous PU foam. Figure $2 \mathrm{c}$ shows the relative change in the current upon application of $300 \mathrm{~Pa}$ pressure; the inset indicates a short response time of $20 \mathrm{~ms}$, which is better than that for previously reported pressure sensors with a similar design but different sensing materials. ${ }^{19,32}$ In addition, in Figure $2 \mathrm{~d}$, the change in current with a continuous increase in pressure from 0.1 to $20 \mathrm{kPa}$ shows stable performance. The response and relaxation curves for the pressure sensor over 10000 loading/ unloading cycles under $1 \mathrm{kPa}$ are shown in Figure 2e. There is no apparent difference in the relative change in current or hysteresis during a large number of iterations. In addition, the pressure sensor attached to the wrist of a male graduate student detected the periodic radial artery pulse, as depicted in Figure $2 \mathrm{f}$. A typical wrist pulse signal has two peaks in general. ${ }^{33}$ Similarly, the measured radial artery pulse exhibits two clearly distinguishable peaks as shown in Figure 2g: systolic and diastolic peaks marked 'a' and 'b,' respectively. Important parameters such as reflection index (RI) and stiffness index (SI) can be calculated as follows: (1) RI=magnitude of diastolic peak (b)/ magnitude of systolic peak (a) $\times 100(\%)$ and (2) arterial SI = subject height $(h) /$ transit time $\left(\Delta T_{\mathrm{DVP}}\right)$. Furthermore, it was reported that the pulse pattern can be changed with health condition and age. ${ }^{24} \Delta T_{\mathrm{DVP}}$ is defined as the time delay between the systolic and diastolic peaks. ${ }^{18}$ The RI and SI are estimated to be $53 \%$ and 7.6, respectively, indicating the healthy state of a 30-year-old man. ${ }^{34}$

Figure 3a schematically shows how the temperature can be monitored with the fabricated MF sensor using the thermoelectric effect. When a temperature gradient is applied to the thermoelectric MWCNT-PANI material, electrons in the higher-temperature region have higher kinetic energy than their Fermi level and diffuse to the lower-temperature region, thus generating a potential difference. ${ }^{35}$ According to this thermoelectric mechanism, the output voltage ( $V_{\text {therm }}$ ) of the temperature sensor is defined as $V_{\text {therm }}=S_{\mathrm{T}} \times \Delta T$, where $S_{\mathrm{T}}$ is the Seebeck coefficient and $\Delta T$ is the temperature gradient on the device. ${ }^{10,36}$ As shown in Figure $3 \mathrm{~b}$, a linear $I-V$ curve is obtained when a temperature gradient is applied. Because of the thermoelectric effect of the MWCNT-PANI nanocomposite, the $I-V$ curve shifts with $V_{\text {therm }}$ for a temperature difference of -20 to $20^{\circ} \mathrm{C}$. Figure $3 \mathrm{c}$ presents the measured output voltage as a function of a temperature difference ranging from -25 to $25^{\circ} \mathrm{C}$. The small error bars correspond to the s.d. of data obtained from five different sensors, 
a

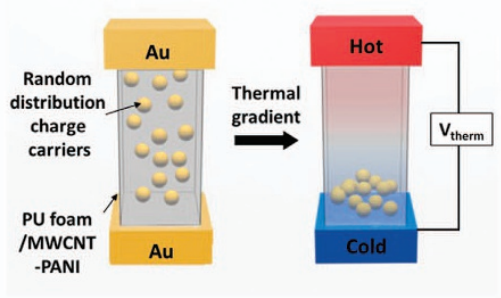

C

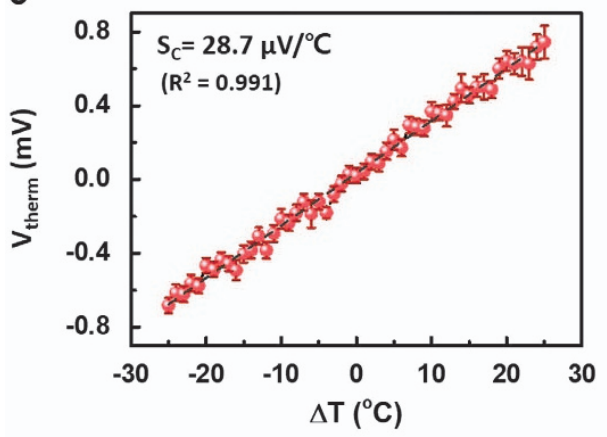

b

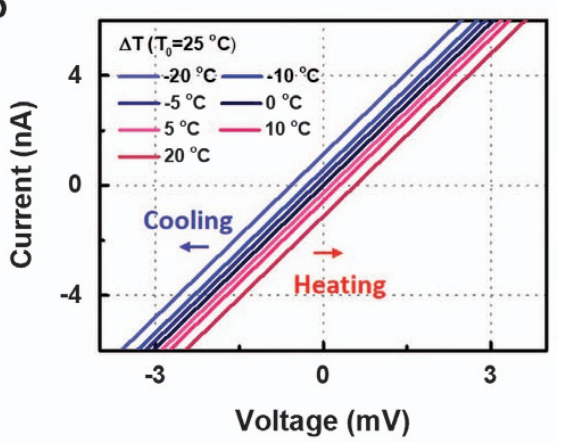

d

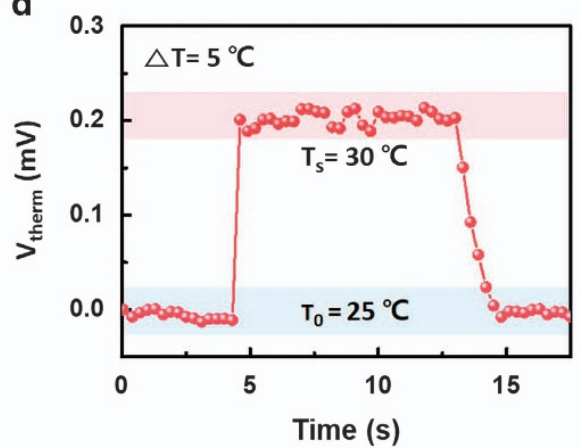

Figure 3 (a) Schematic of output voltage $\left(V_{\text {therm }}\right)$ generated owing to a temperature gradient $(\Delta T)$. (b) $I-V$ characteristics of the temperature sensor with variation in $\Delta T$. (c) $V_{\text {therm }}$ vs $\Delta T$. (d) Response curve with a temperature stimulus of $5^{\circ} \mathrm{C}$, showing a response time of $300 \mathrm{~ms}$.

showing high reproducibility. The output voltage change exhibits a linear dependence on the temperature difference. The Seebeck coefficient $\left(S_{\mathrm{T}}\right)$ is estimated to be $28.7 \mu \mathrm{V} /{ }^{\circ} \mathrm{C}^{-1}\left(R^{2}=0.991\right)$ via a linear least-squares fitting. The $S_{\mathrm{T}}$ of PANI, $2.98 \mu \mathrm{V} /{ }^{\circ} \mathrm{C}^{-1}$, is much lower than that of the MWCNT-PANI nanocomposite, as shown in Supplementary Figure S7b. Introduction of CNTs to PANI enhances the electrical conductivity of the composite by facilitating the interaction between PANI chains and CNTs through charge transfer. ${ }^{12}$ The temperature sensor exhibits response and relaxation times of $300 \mathrm{~ms}$ and $1.2 \mathrm{~s}$, respectively, for a temperature difference of $5{ }^{\circ} \mathrm{C}$, which are better than those of previously reported temperature sensors (Figure 3d). ${ }^{10,26}$ The short response time is attributed to high thermal diffusivity and low heat capacity owing to the use of the MWCNTPANI composite. ${ }^{35}$

The fabrication of self-powered MF sensing devices is a challenge in the development of sustainable flexible sensors. In principle, the pressure applied to the sensor can be measured without any additional power supply because of the thermoelectric temperature-sensing mechanism. Supplementary Figure S7c illustrates that both temperature and pressure can be simultaneously applied to the sensor to achieve electrical changes in the $I-V$ curve. Of note, the temperature stimulation has a limited effect on the electrical resistance, and the pressure signal shows a negligible effect on $V_{\text {therm. }}$. These properties can be represented by the voltage and current signals upon application of a temperature and pressure difference, respectively. A corresponding current response to a fixed pressure is observed when the temperature gradient is varied between 1 and $10^{\circ} \mathrm{C}$, as shown in Figure $4 \mathrm{a}$ and $\mathrm{b}$. Although the output voltage is generated owing to the temperature gradient, the current is also generated simultaneously. Thus, in Figure $4 \mathrm{a}$, the initial current changes as the temperature gradient varies. However, the normalized current was observed to correspond to the value for specific pressure regardless of the temperature gradient, as shown in Figure $4 \mathrm{~b}$. This finding suggests that the signals from the MF sensors, both temperature and pressure, are measured simultaneously without any interference. To evaluate the self-power and simultaneous operation of the MF sensor, the current change and the output voltage response were monitored for loading/ unloading cycles of pressure and temperature, respectively (Figure $4 \mathrm{c}$ and $\mathrm{d}$ ). The MF sensor showed reproducible current change and output voltage in response to a fixed pressure of $1 \mathrm{kPa}$ and temperature of $20^{\circ} \mathrm{C}$. The device simultaneously recognized a slight pressure difference between fingertip touches of $\sim 15$ and $\sim 30 \mathrm{kPa}$ and the temperature of the fingertip $\left(31.6^{\circ} \mathrm{C}\right.$, Supplementary Figure S8a). The pressure and temperature were calibrated using the pressure- and temperature-dependent behavior of the current and output voltage, respectively, shown in Figures $2 \mathrm{~b}$ and $3 \mathrm{c}$. Thus it was confirmed that the temperature and pressure can be measured as dual parameters by the generated output voltage and current, respectively, without any interference.

Ammonia $\left(\mathrm{NH}_{3}\right)$ is one of the most harmful environmental pollutants, and exposure to large quantities of $\mathrm{NH}_{3}$ (concentrations $>300$ p.p.m.) can cause serious damage to human cells, leading to injury of the skin, eyes and respiratory tract. ${ }^{37} \mathrm{NH}_{3}$ is also a natural byproduct of various metabolic activities in the human body. ${ }^{38}$ Hence, detection of $\mathrm{NH}_{3}$ is very important in terms of environmental and health monitoring.

A resistor-type sensor of PU foam coated with MWCNT-PANI nanocomposite can be used to detect $\mathrm{NH}_{3}$ by monitoring changes in the resistance upon adsorption of $\mathrm{NH}_{3}$ molecules inside a glass tube chamber at $25^{\circ} \mathrm{C}$. With an increase in $\mathrm{NH}_{3}$ exposure, the resistance increases but recovers the initial value after flushing with $\mathrm{N}_{2}$ gas, as shown in Figure 5a. Figure 5b shows the response time of $7.8 \mathrm{~s}$ and recovery time of $34 \mathrm{~s}$, observed under exposure to 20 p.p.m. $\mathrm{NH}_{3}$ at room temperature, which are shorter than those for an MWCNT- 

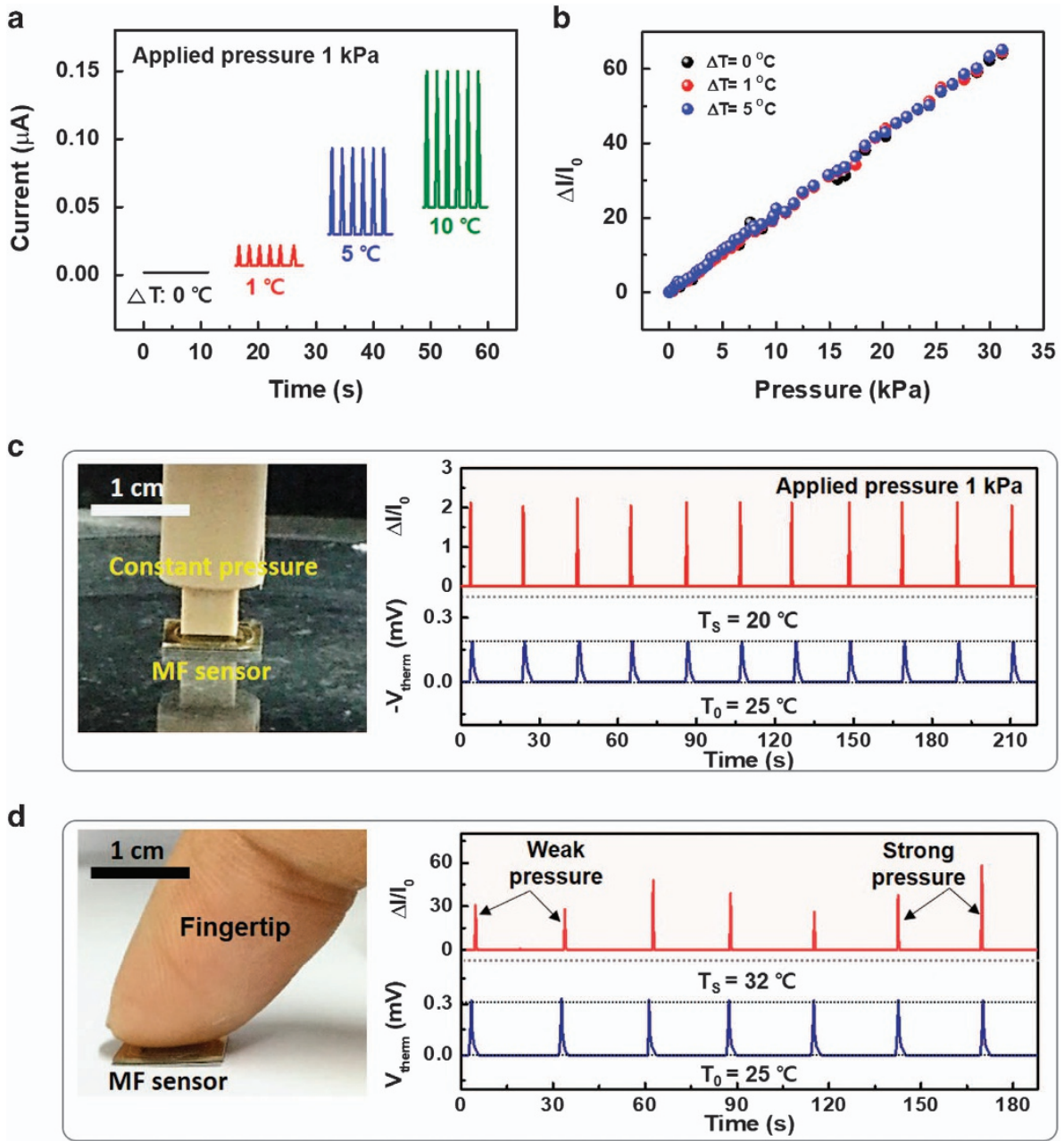

Figure 4 (a) Change in current from the MF sensor at various temperature gradients $\left(0,1,5\right.$ and $\left.10^{\circ} \mathrm{C}\right)$ under a constant pressure of $1 \mathrm{kPa}$. (b) Current response $\left(\Delta / / I_{0}\right)$ of the MF sensor to various pressures under different temperature gradients $(\Delta T) . \Delta / / I_{0}$ and $V_{\text {therm }}$ of the MF sensor in response to (c) loading/unloading cycles at a constant pressure of $1 \mathrm{kPa}$ and (d) fingertip touch; $T_{0}$ is an ambient temperature of $25^{\circ} \mathrm{C}$.

based $\mathrm{NH}_{3}$ gas sensor (Supplementary Figure $\mathrm{S9a}$ and b). The response and recovery times of the $\mathrm{NH}_{3}$ sensor based on the bare MWCNTs were calculated to be 900 and 2410 s, respectively, which are much longer than those of the sensor based on the MWCNTPANI nanocomposite. Further, the long recovery time of the bare MWCNTs may be due to gas molecules entrapped in the inner tubes of the MWCNTs and their bonding with the defects. The enhanced sensing performance of the MWCNT-PANI nanocomposite might be due to the combined effect of doping/de-doping of PANI and the electron transfer between the $\mathrm{NH}_{3}$ molecules and the MWCNTs. ${ }^{12}$ $\mathrm{NH}_{3}$ molecules are electron donors and hence act as dopants of p-type PANI. $\mathrm{NH}_{3}$ molecules adsorbed onto the surface of PANI react with the $\mathrm{N}-\mathrm{H}$ groups of PANI to form $\mathrm{NH}_{4}{ }^{+}\left(\mathrm{NH}_{3}\right.$ ions). This increases the resistance of the sensor by detecting the polaron of PANI. During this reaction, PANI is converted into emeraldine (base form). The emeraldine salt form can be recovered by desorption of the $\mathrm{NH}_{3}$ molecules upon flushing with $\mathrm{N}_{2}$. During this reversible reaction, $\mathrm{NH}_{4}{ }^{+}$ions decompose to $\mathrm{NH}_{3}$ and a proton, and therefore, the base resistance can be recovered, and PANI is de-doped. ${ }^{39,40}$

Supplementary Table S1 shows that the sensor performance in this work is superior to the performance of previously reported $\mathrm{NH}_{3}$ gas sensors based on different sensing materials. Supplementary Figure S10 depicts the band diagram of the MWCNT-PANI nanocomposites and corresponding electron transfer. Electrons generated by the $\mathrm{NH}_{3}$ molecules adsorbed onto PANI can be easily transferred to MWCNTs and then to the Au electrodes according to the energy bands. ${ }^{40-43}$

In the core-shell-type structure, where PANI is completely coated on the surface of MWCNTs, as evident from Figure 1b, the charge transfer between PANI and the MWCNTs is enhanced owing to the strong interfacial affinity. The MWCNTs most likely withdraw electrons from the reduced PANI, leading to complete recovery of the gas sensor based on the MWCNT-PANI nanocomposite within a short time. ${ }^{44}$

Supplementary Figure S11a shows the real-time cyclic response of the gas sensor to 25 p.p.m. $\mathrm{NH}_{3}$ gas. The response time of the sensor ranged from approximately 7 to $10 \mathrm{~s}$ with variation in the $\mathrm{NH}_{3}$ concentration, as shown in Supplementary Figure S11b. The recovery time increased rapidly with increased exposure. As seen in Figure $5 c$, the gas sensor shows a linear sensitivity to $\mathrm{NH}_{3}$ concentrations ranging from 5 to 200 p.p.m., even considering the error bars $\left(R^{2}=0.994\right)$. Above exposure to 200 p.p.m. $\mathrm{NH}_{3}$, the sensitivity seems to be nearly saturated regardless of the increase in concentration. The sensitivity is a normalized change in resistance, $\left(R_{\mathrm{NH}_{3}}-R_{0}\right) / R_{0} \times 100(\%)$ upon exposure to $\mathrm{NH}_{3}$ gas, where $R_{\mathrm{NH}_{3}}$ and $R_{0}$ are the resistance with and without $\mathrm{NH}_{3}$ gas, respectively. The selectivity of the sensor was 
a

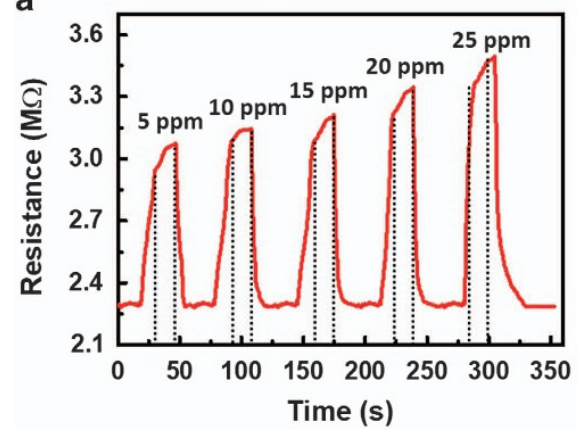

C

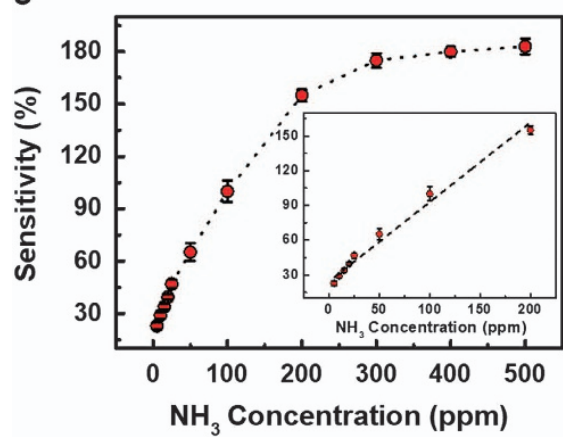

b

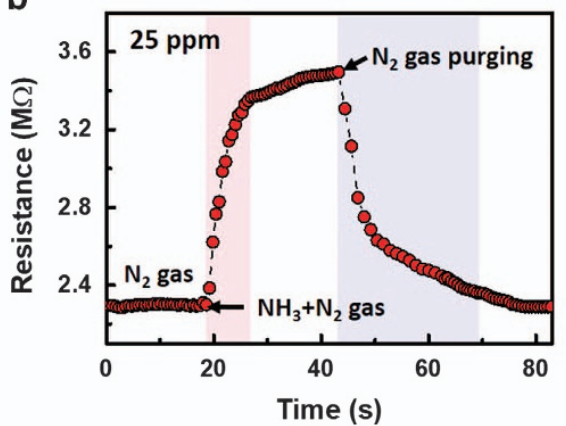

d

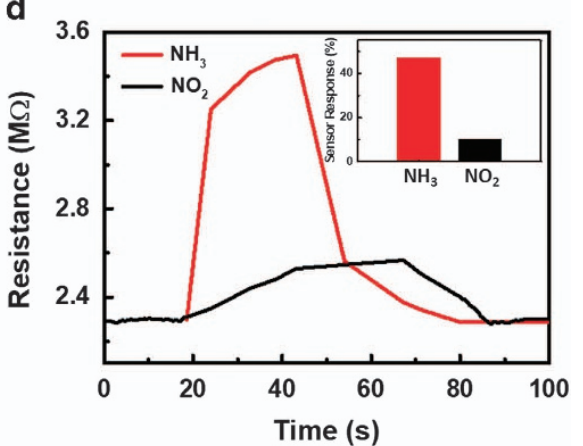

Figure 5 (a) Response of the sensor to various $\mathrm{NH}_{3}$ concentrations diluted in a $\mathrm{N}_{2}$ gas flow. (b) Response and recovery of the gas sensor exposed to 25 p.p.m. $\mathrm{NH}_{3}$ at room temperature. (c) Sensitivity to variations in $\mathrm{NH}_{3}$ concentration. (d) Response and recovery of a gas sensor exposed to 25 p.p.m. $\mathrm{NH}_{3}$ (red) and $\mathrm{NO}_{2}$ (black) gases at room temperature. The inset shows the sensitivity of a sensor to 25 p.p.m. $\mathrm{NH}_{3}\left(\right.$ red) and $\mathrm{NO}_{2}$ (black) gases at room temperature.

measured at room temperature with the two environmentally toxic gases $\mathrm{NH}_{3}$ and $\mathrm{NO}_{2}$ at the same concentration (25 p.p.m.). As is clearly shown in Figure $5 \mathrm{~d}$, higher sensitivity to $\mathrm{NH}_{3}$ was observed. The figure inset shows that the sensitivity to $\mathrm{NH}_{3}$ and $\mathrm{NO}_{2}$ gases was estimated to be $46.9 \%$ and $10.2 \%$, respectively, demonstrating selectivity for $\mathrm{NH}_{3}$ over $\mathrm{NO}_{2}$.

Next, a stretchable bio-environmental sensor system consisting of $5 \times 5 \mathrm{MF}$ sensors and a $\mathrm{NH}_{3}$ gas sensor was fabricated via using a cutting machine and thermal release tape (Figure 6). The detailed dimensions of the stretchable sensor array are depicted in Supplementary Figure S12. For simultaneous measurement of spatially resolved temperature and pressure mapping under deformation on the skin, the stretchable MF sensor array was attached to the skin on the hand. By using the mixed thin PDMS and Silbione film, strong adhesion onto the skin is guaranteed, and the adhesiveness was maintained with repetitive attaching/detaching cycles even after washing in water (Supplementary Figure S13). Among adhesive elastomers, Silbione, a commercial soft skin adhesive gel, deforms easily to make conformal contact with non-planar surfaces due to its viscoelasticity, which has an important role in the performance of soft pressure-sensitive adhesives. ${ }^{45}$ Furthermore, Silbione has long been recognized as a biocompatible and effective material for a variety of wound and skin care applications, including wound dressing bandages and scar treatment. ${ }^{46}$

Strain distribution on the sensor array under stretching was analyzed using the finite element method. Commercial software, ABAQUS, was used to model the stretchable system. The neoHookean constitutive model was used for the mixture of PDMS and Silbione substrate. An optical image of the stretchable MF sensor array under biaxial strain of $50 \%$ is shown in Figure 6 a. Figure $6 \mathrm{~b}$ shows the results of the finite element method simulation under the same biaxial strain. Here the applied biaxial strain is defined as $\varepsilon_{\text {applied }}=\left(l-l_{0}\right) \times 100 / l_{0}$, where $l_{0}$ and $l$ are the lengths of a unit module before and after stretching, respectively (Figure 6c). Under biaxial stretching of $50 \%$, the strain between adjacent PET films was estimated to be $198.7 \%$ and $217.6 \%$ in the horizontal and longitudinal directions, respectively, consistent with the experimentally measured strain values shown in Figure $6 \mathrm{~b}$. Because of the stress singularity at the interface corner between the PET film and the substrate, the strain near the corner of the PET film was much higher than that in the surrounding region. 47

The fabricated MF sensor exhibited stable performance under 5000 repetitive biaxial stretching/releasing cycles up to $50 \%$, as is clearly shown in Figure $6 \mathrm{~d}$ and e. The normalized current $\left(I / I_{0}\right)$ due to an applied pressure of $5 \mathrm{kPa}$ and the output voltage $\left(V_{\text {therm }} / V_{\text {therm0 } 0}\right)$ due to a temperature change of $5{ }^{\circ} \mathrm{C}$ remained almost the same, regardless of the applied strain. Here, $I$ and $I_{0}$ correspond to the current under pressure after and before stretching, respectively, and $V_{\text {therm }}$ and $V_{\text {therm0 }}$ are the output voltage after and before stretching, respectively. Even after 5000 cycles, the variation was within 6\%. These results clearly suggest that our MF sensor on a stretchable substrate is very stable under repetitive biaxial stretching.

The fingertip, one of the most sensitive parts of the human skin, can distinguish temperature and pressure stimuli with high spatial resolution. ${ }^{10}$ When a human fingertip touches a dummy fingertip on which the fabricated sensor array is attached, the corresponding pressure and temperature mapping can be obtained via measurement of the normalized change in the current and output voltage, respectively (Figure 6f). The pressure and temperature are estimated using the pressure- and temperature-dependent behavior of the current and output voltage, respectively, as shown in Figures $2 \mathrm{~b}$ and $3 c$. The fingertip temperature measured using an infrared radiation 
a

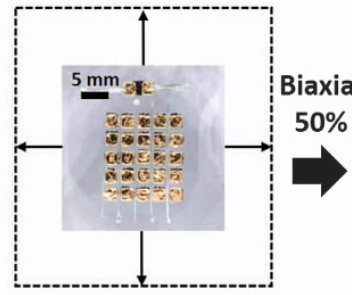

C

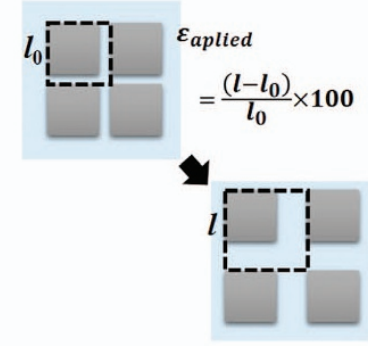

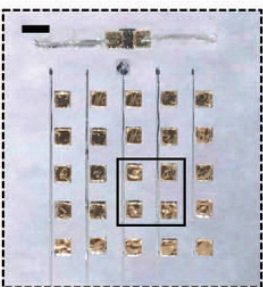

d

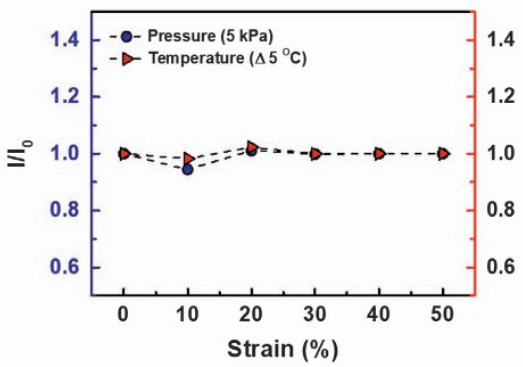

b

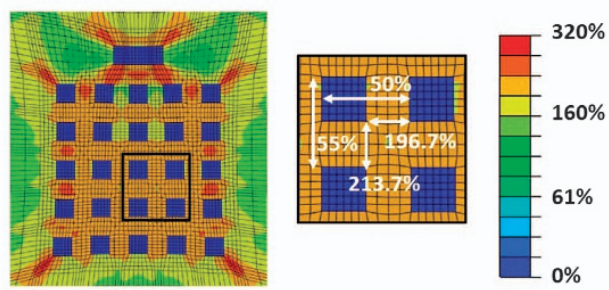

e f

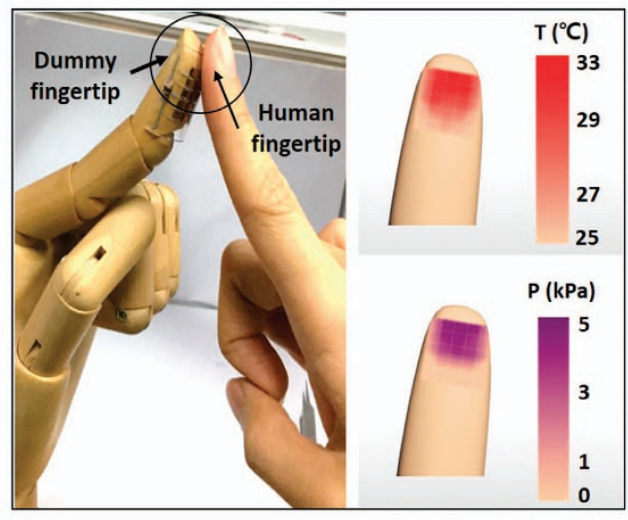

$\mathrm{g}$

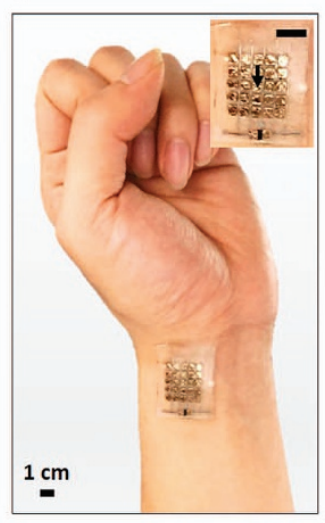

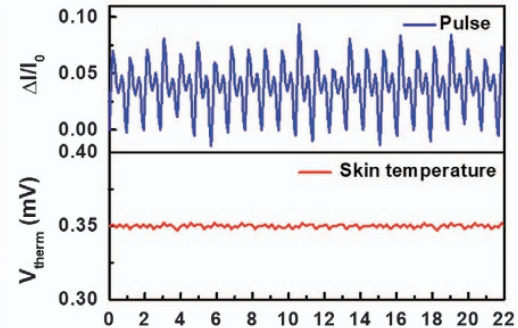

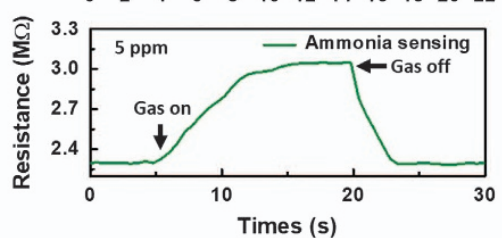

Figure 6 (a) Optical images and (b) strain distribution of the stretchable MF sensor array upon biaxial stretching of 50\%. The insets in panels (a) and (b) show an enlarged optical image and FEM analysis. (c) Definition of applied biaxial strain on the stretchable substrate. (d) Normalized current $\left(/ / I_{0}\right)$ and output voltage $\left(V_{\text {therm }} / V_{\text {thermo }}\right)$ under an applied pressure and temperature gradient of $5 \mathrm{kPa}$ and $5{ }^{\circ} \mathrm{C}$ vs biaxial strain. Here $I$ and $I_{0}$ correspond to the current under a pressure of $5 \mathrm{kPa}$ after and before stretching, respectively. $V_{\text {therm }}$ and $V_{\text {thermo }}$ are the output voltage at $\Delta \mathrm{T}=5^{\circ} \mathrm{C}$ after and before stretching, respectively. (e) $I / I_{0}$ and $V_{\text {therm }} / V_{\text {thermo }}$ as a function of stretching cycles under a strain of $50 \%$. (f) Photograph of a wearable MF sensor array on a prosthetic hand touching a human fingertip (left). Temperature and pressure mapping of the sensing array (right). (g) Photograph of wearable MF sensor array on wrist (left). Plot of current, output voltage and resistance due to pulse, skin temperature and ammonia gas sensing, respectively (right). This plot was constructed from measurements of the sensor in the $(3,3)$ pixel.

thermometer was $31.6^{\circ} \mathrm{C}$ (Supplementary Figure S8a), which is close to that measured by our temperature sensor, $32^{\circ} \mathrm{C}$. These results demonstrate the excellent dual parameter sensing capability of our MF sensor array. Furthermore, after contacting a tiny ice cube with a contact area of $3.5 \times 3.5 \mathrm{~mm}^{2}$ (4 pixels) with an MF sensor array attached to the back of the hand, the spatially resolved pressure $(4-5 \mathrm{kPa})$ and temperature $\left(0-5{ }^{\circ} \mathrm{C}\right)$ information could be collected on a reconstructed map, as shown in Supplementary Figure S14a.

The stretchable bio-environmental sensor array was demonstrated by attaching it to the human wrist, as shown in Figure $6 \mathrm{~g}$. The resistor-type $\mathrm{NH}_{3}$ gas sensor was electrically separated for independent detection from the array of pressure/temperature sensors, as shown in Supplementary Figure S15. Time-dependent changes in the normalized current show the highest measured pulse pressure among the 25 sensors. The highest value at pixel $(3,3)$ is seen in Supplementary Figure S14b and c. In addition, as the stretchable MF sensor array was attached to the skin as a whole, it was confirmed that the temperature difference measured by the 25 sensors was $8 \pm 0.04{ }^{\circ} \mathrm{C}$. Here $I_{0}$ and $I$, respectively, denote the currents measured before and after the application of pressure by a pulse. Thus the skin temperature was estimated to be $33^{\circ} \mathrm{C}$ considering the room temperature of $25^{\circ} \mathrm{C}$, which is close to the wrist temperature of $33.4^{\circ} \mathrm{C}$ measured with an infrared radiation thermometer (Supplementary Figure S8b). With an integrated gas sensor, hazardous $\mathrm{NH}_{3}$ gas can be successfully detected at a concentration of 5 p.p.m. These results demonstrate the potential application of our MF sensor array as a skin-attached device that can simultaneously detect pressure, temperature and $\mathrm{NH}_{3}$ gas even under deformation due to body movements. 


\section{CONCLUSION}

We have demonstrated a skin-like stretchable array of highperformance bio-environmental sensors developed via material design and a novel device fabrication strategy. In particular, the combination of PU foam with a microporous structure and the MWCNT-PANI nanocomposite with high electrical conductivity and good thermoelectric properties enables simultaneous detection of pressure, temperature and $\mathrm{NH}_{3}$ gas with high sensitivity. Furthermore, the facile fabrication of the stretchable device facilitates application to e-skin and skin-attachable health monitoring systems. ${ }^{48}$

\section{CONFLICT OF INTEREST}

The authors declare no conflict of interest.

\section{ACKNOWLEDGEMENTS}

This work was supported by a National Research Foundation of Korea (NRF) grant funded by the Korea government (MEST) (grant no.

NRF-2016R1A2A1A05004935). This work was also partially supported by an Institute for Information and Communications Technology Promotion (IITP) grant funded by the Korea government (MSIP) (B0101-16-0133, the core technology development of light and space adaptable energy saving I/O platform for future advertising service). This work was supported by the KUKIST School Project.

\section{PUBLISHER'S NOTE}

Springer Nature remains neutral with regard to jurisdictional claims in published maps and institutional affiliations.

1 Webb, R. C., Bonifas, A. P., Behnaz, A., Zhang, Y., Yu, K. J., Cheng, H., Shi, M. Bian, Z., Liu, Z., Kim, Y.-S., Yeo, W.-H., Park, J. S., Song, J., Li, Y., Huang, Y., Gorbach, A. M. \& Rogers, J. A. Ultrathin conformal devices for precise and continuous thermal characterization of human skin. Nat. Mater. 12, 938-944 (2013).

2 Kim, J., Lee, M., Shim, H. J., Ghaffari, R., Cho, H. R., Son, D., Jung, Y. H., Soh, M., Choi, C., Jung, S., Chu, K., Jeon, D., Lee, S.-T., Kim, J. H., Choi, S. H., Hyeon, T. \& Kim, D.-H. Stretchable silicon nanoribbon electronics for skin prosthesis. Nat Commun. 5, 5747 (2014).

3 Chen, L. Y., Tee, B. C. K., Chortos, A. L., Schwartz, G., Tse, V., Lipomi, D. J., Wong, H. S. P., McConnell, M. V. \& Bao, Z. Continuous wireless pressure monitoring and mapping with ultra-small passive sensors for health monitoring and critical care. Nat. Commun. 5, 5028 (2014)

4 Kim, J., Kim, M., Lee, M.-S., Kim, K., Ji, S., Kim, Y.-T., Park, J., Na, K., Bae, K.-H. Kim, H. K., Bien, F., Lee, C.Y. \& Park, J.-U. Wearable smart sensor systems integrated on soft contact lenses for wireless ocular diagnostics. Nat. Commun. 8, 14997 (2017).

5 Takei, K., Takahashi, T., Ho, J. C., Ko, H., Gillies, A. G., Leu, P. W., Fearing, R. S. \& Javey, A. Nanowire active-matrix circuitry for low-voltage macroscale artificial skin. Nat. Mater. 9, 821-826 (2010)

6 Choi, S., Lee, H., Ghaffari, R., Hyeon, T. \& Kim, D.-H. Recent advances in flexible and stretchable bio-electronic devices integrated with nanomaterials. Adv. Mater. 28, 4203-4218 (2016).

7 Wang, X., Dong, L., Zhang, H., Yu, R., Pan, C. \& Wang, Z. L. Recent progress in electronic skin. Adv. Sci. 2, 1500169 (2015).

8 Liao, X., Liao, Q., Zhang, Z., Yan, X., Liang, Q., Wang, Q., Li, M. \& Zhang, Y. A highly stretchable ZnO@fiber-based multifunctional nanosensor for strain/temperature/UV detection. Adv. Funct. Mater. 26, 3074-3081 (2016).

9 Yamamoto, Y., Harada, S., Yamamoto, D., Honda, W., Arie, T., Akita, S. \& Takei, K. Printed multifunctional flexible device with an integrated motion sensor for health care monitoring. Sci. Adv. 2, e1601473 (2016).

10 Zhang, F., Zang, Y., Huang, D., Di, C.-a. \& Zhu, D. Flexible and self-powered temperature-pressure dual-parameter sensors using microstructure-frame-supported organic thermoelectric materials. Nat. Commun. 6, 8356 (2015).

11 Kraemer, D., Poudel, B., Feng, H.-P., Caylor, J. C., Yu, B., Yan, X., Ma, Y., Wang, X. Wang, D., Muto, A., McEnaney, K., Chiesa, M., Ren, Z. \& Chen, G. High-performance flat-panel solar thermoelectric generators with high thermal concentration. Nat. Mater. 10, 532-538 (2011)

12 Abdulla, S., Mathew, T. L. \& Pullithadathil, B. Highly sensitive, room temperature gas sensor based on polyaniline-multiwalled carbon nanotubes (PANI/MWCNTs) nanocomposite for trace-level ammonia detection. Sens. Actuators B 221, 1523-1534 (2015).

13 Kar, P. \& Choudhury, A. Carboxylic acid functionalized multi-walled carbon nanotube doped polyaniline for chloroform sensors. Sens. Actuators B 183, 25-33 (2013).
14 Chen, K., Gao, W., Emaminejad, S., Kiriya, D., Ota, H., Nyein, H. Y. Y., Takei, K. \& Javey, A. Printed carbon nanotube electronics and sensor systems. Adv. Mater. 28, 4397-4414 (2016)

15 Park, H., Jeong, Y. R., Yun, J., Hong, S. Y., Jin, S. W., Lee, S.-J., Zi, G. \& Ha, J. S. Stretchable array of highly sensitive pressure sensors consisting of polyaniline nanofibers and Au-coated polydimethylsiloxane micropillars. ACS Nano 9, 9974-9985 (2015).

16 Sobha, A. P. \& Narayanankutty, S. K. Electrical and thermoelectric properties of functionalized multiwalled carbon nanotube/polyaniline composites prepared by different methods. IEEE Trans. Nanotechnol. 13, 835-841 (2014).

17 Meng, C., Liu, C. \& Fan, S. A promising approach to enhanced thermoelectric properties using carbon nanotube networks. Adv. Mater. 22, 535-539 (2010).

18 Choong, C. L., Shim, M. B., Lee, B. S., Jeon, S., Ko, D. S., Kang, T. H., Bae, J., Lee, S. H., Byun, K. E., Im, J., Jeong, Y. J., Park, C. E., Park, J.-J. \& Chung, U.-I. Highly stretchable resistive pressure sensors using a conductive elastomeric composite on a micropyramid array. Adv. Mater. 26, 3451-3458 (2014).

19 Zhong, W., Liu, Q., Wu, Y., Wang, Y., Qing, X., Li, M., Liu, K., Wang, W. \& Wang, D. A nanofiber based artificial electronic skin with high pressure sensitivity and 3D conformability. Nanoscale 8, 12105-12112 (2016).

20 Gong, S., Schwalb, W., Wang, Y., Chen, Y., Tang, Y., Si, J., Shirinzadeh, B. \& Cheng, W. A wearable and highly sensitive pressure sensor with ultrathin gold nanowires. Nat. Commun. 5, 3132 (2014).

21 Han, J.-W., Kim, B., Li, J. \& Meyyappan, M. Flexible, compressible, hydrophobic, floatable, and conductive carbon nanotube-polymer sponge. Appl. Phys. Lett. 102, 051903 (2013).

22 Yavari, F., Chen, Z., Thomas, A. V., Ren, W., Cheng, H.-M. \& Koratkar, N. High sensitivity gas detection using a macroscopic three-dimensional graphene foam network. Sci. Rep. 1, 166 (2011)

23 Gong, S., Zhao, Y., Yap, L. W., Shi, Q., Wang, Y., Bay, J. A. P. B., Lai, D. T. H. Uddin, H. \& Cheng, W. Fabrication of highly transparent and flexible nanomesh electrode via self-assembly of ultrathin gold nanowires. Adv. Electron. Mater. 2, 1600121 (2016).

24 Franklin, S., Gustin, W., Wong, N. D., Larson, M. G., Weber, M. A., Kannel, W. B. \& Levy, D. Hemodynamic patterns of age-related changes in blood pressure. Circulation 96, 308-315 (1997).

25 Niu, H., Qin, S., Mao, X., Zhang, S., Wang, R., Wan, L., Xu, J. \& Miao, S. Axle-sleeve structured MWCNTs/polyaniline composite film as cost-effective counter-electrodes for high efficient dye-sensitized solar cells. Electrochim. Acta 121, 285-293 (2014).

26 Konyushenko, E. N., Stejskal, J., Trchová, M., Hradil, J., Kovářová, J., Prokeš, J., Cieslar, M., Hwang, J.-Y., Chen, K.-H. \& Sapurina, I. Multi-wall carbon nanotubes coated with polyaniline. Polymer 47, 5715-5723 (2006).

27 Hong, S. Y., Lee, Y. H., Park, H., Jin, S. W., Jeong, Y. R., Yun, J., You, I., Zi, G. \& Ha, J. S. Stretchable active matrix temperature sensor array of polyaniline nanofibers for electronic skin. Adv. Mater. 28, 930-935 (2015).

28 Hong, S. Y., Yoon, J., Jin, S. W., Lim, Y., Lee, S.-J., Zi, G. \& Ha, J. S. High-density, stretchable, all-solid-state microsupercapacitor arrays. ACS Nano $\mathbf{8}$ 8844-8855 (2014).

29 Boley, J. W., White, E. L., Chiu, G. T. C. \& Kramer, R. K. Direct writing of gallium-indium alloy for stretchable electronics. Adv. Funct. Mater. 24, 3501-3507 (2014).

30 Doudrick, K., Shanliangzi, L., Mutunga, E. M., Klein, K. L., Damle, V., Varanasi, K. K. \& Rykaczewski, K. Different shades of oxide: from nanoscale wetting mechanisms to contact printing of gallium-based liquid metals. Langmuir 30, 6867-6877 (2014).

31 Jeong, S., Hjort, K. \& Wu, Z. Tape transfer printing of a liquid metal alloy for stretchable RF electronics. Sensors 14, 16311 (2014).

32 Yao, H.-B., Ge, J., Wang, C.-F., Wang, X., Hu, W., Zheng, Z.-J., Ni, Y. \& Yu, S.-H. A flexible and highly pressure-sensitive graphene-polyurethane sponge based on fractured microstructure design. Adv. Mater. 25, 6692-6698 (2013).

33 Rangaprakash, D. \& Narayana Dutt, D. Study of wrist pulse signals using time domain spatial features. Comput. Electrical Eng. 45, 100-107 (2015).

34 Simek, J., Wichterle, D., Melenovsky, V., Malik, J., Svobodova, J. \& Svacina, S. Pulse wave analysis during supine rest may identify subjects with recurrent vasovagal syncope. Clin. Sci. 109, 165-170 (2005).

35 Borup, K. A., de Boor, J., Wang, H., Drymiotis, F., Gascoin, F., Shi, X., Chen, L., Fedorov, M. I., Muller, E., Iversen, B. B. \& Snyder, G. J. Measuring thermoelectric transport properties of materials. Energy Environ. Sci. 8, 423-435 (2015).

36 Venkatasubramanian, R., Siivola, E., Colpitts, T. \& O'Quinn, B. Thin-film thermoelectric devices with high room-temperature figures of merit. Nature 413, 597-602 (2001).

37 Wu, Z., Chen, X., Zhu, S., Zhou, Z., Yao, Y., Quan, W. \& Liu, B. Enhanced sensitivity of ammonia sensor using graphene/polyaniline nanocomposite. Sens. Actuators B 178 , 485-493 (2013).

38 Timmer, B., Olthuis, W. \& Berg, A. v. d. Ammonia sensors and their applicationsa review. Sens. Actuators B 107, 666-677 (2005).

39 He, L., Jia, Y., Meng, F., Li, M. \& Liu, J. Gas sensors for ammonia detection based on polyaniline-coated multi-wall carbon nanotubes. Mater. Sci. Eng. B 163 , 76-81 (2009).

40 Sharma, S., Hussain, S., Singh, S. \& Islam, S. S. MWCNT-conducting polymer composite based ammonia gas sensors: a new approach for complete recovery process. Sens. Actuators B 194, 213-219 (2014).

$41 \mathrm{Li}$, D., Huang, J. \& Kaner, R. B. Polyaniline nanofibers: a unique polymer nanostructure for versatile applications. Acc. Chem. Res. 42, 135-145 (2009).

42 Salvatierra, R. V., Oliveira, M. M. \& Zarbin, A. J. G. One-pot synthesis and processing of transparent, conducting, and freestanding carbon nanotubes/polyaniline composite films. Chem. Mater. 22, 5222-5234 (2010). 
43 Xue, L., Wang, W., Guo, Y., Liu, G. \& Wan, P. Flexible polyaniline/carbon nanotube nanocomposite film-based electronic gas sensors. Sens. Actuators B 244, 47-53 (2017).

44 Ding, M., Tang, Y., Gou, P., Reber, M. J. \& Star, A. Chemical sensing with polyaniline coated single-walled carbon nanotubes. Adv. Mater. 23, 536-540 (2011)

45 Patil, S., Malasi, A., Majumder, A., Ghatak, A. \& Sharma, A. Reusable antifouling viscoelastic adhesive with an elastic skin. Langmuir 28, 42-46 (2012).

46 Jang, K.-I., Chung, H. U., Xu, S., Lee, C. H., Luan, H., Jeong, J., Cheng, H., Kim, G.-T., Han, S. Y., Lee, J. W., Kim, J., Cho, M., Miao, F., Yang, Y., Jung, H. N., Flavin, M., Liu, H., Kong, G. W., Yu, K. J., Rhee, S. I., Chung, J., Kim, B., Kwak, J. W., Yun, M. H., Kim, J. Y., Song, Y. M., Pail, U., Zhang, Y., Huang, Y. \& Rogers, J. A. Soft network composite materials with deterministic and bio-inspired designs. Nat. Commun. 6, 6566 (2015)

47 Yoon, J., Hong, S. Y., Lim, Y., Lee, S. J., Zi, G. \& Ha, J. S. Design and fabrication of novel stretchable device arrays on a deformable polymer substrate with embedded liquid-metal interconnections. Adv. Mater. 26, 6580-6586 (2014).

48 Yang, S., Chen, Y.-C., Nicolini, L., Pasupathy, P., Sacks, J., Su, B., Yang, R., Sanchez, D., Chang, Y.-F., Wang, P., Schnyer, D., Neikirk, D. \& Lu, N. "Cut-and- paste" manufacture of multiparametric epidermal sensor systems. Adv. Mater. 27, $6423(2015)$

(c) (i) This work is licensed under a Creative Commons Attribution 4.0 International License. The images or other third party material in this article are included in the article's Creative Commons license, unless indicated otherwise in the credit line; if the material is not included under the Creative Commons license, users will need to obtain permission from the license holder to reproduce the material. To view a copy of this license, visit http:// creativecommons.org/licenses/by/4.0/

(C) The Author(s) 2017

Supplementary Information accompanies the paper on the NPG Asia Materials website (http://www.nature.com/am) 